\title{
Stochastic Automata Network Based Approach for Performance Evaluation of Network-on-Chip Communication Architecture
}

\author{
Ulhas Deshmukh \\ Deptt. of ECE, Govt. Polytechnic, Dhule, India \\ Email:deshmukhur@gmail.com
}

\author{
Vineet Sahula, Senior Member, IEEE \\ Deptt. of ECE, NIT, Jaipur, India \\ Email: sahula@ieee.org
}

\begin{abstract}
Concurrent communication architectures are essential in order to meet ever increasing demand for higher performance of modern-day System-on-Chip (SoC) applications. The behavior of such communication architectures is usually complex and difficult to model. This paper presents a formal modeling approach based on Stochastic Automata Network (SAN) for efficient performance evaluation of concurrent communication architectures. We use functional and synchronizing transitions of the SAN model to describe interaction among concurrent components of these architectures. We propose model for Network-on-Chip (NoC) architecture, and our modeling approach is able to provide evaluation of performance parameters viz. throughput and rate of accepted traffic for mesh. Torus and butterfly Fat Tree topologies. The proposed modeling approach is not only efficient and accurate but also requires lesser modeling efforts.
\end{abstract}

\section{Index Terms}

Stochastic automata network, performance evaluation, $\mathrm{NoC}$

\section{Introduction}

In order to meet ever increasing demand for more functions and higher performance in diverse and emerging applications like telecommunication and multimedia, designers not only use a number of heterogeneous, concurrent Processing Elements (PEs) or Intellectual Property (IP) blocks but also concurrent communication techniques in a single System-on-Chip (SoC). This makes SoC and its design quite complex. Further, rapid technology changes shorten product life

This research was supported by a grant from Ministry of Comm. \& IT, Govt. of India under project SMDP-VLSI-2. cycles and make time-to-market (TTM) a critical issue for designers. Time-to-market consists of time spent in designing, testing, manufacturing and packaging. Manufacturing and packaging times are measured in weeks with low uncertainty, whereas design and verification times are measured in months or years with high uncertainty. Hence, in order to achieve smaller design time and shorter TTM, SoC design flow needs to be analyzed and optimized for design time.

Reuse of pre-designed and pre-verified hardware/software IPs is the way to manage increased complexity and time deadlines of SoCs. Now-a-days fully optimized IPs are available. In SoCs, integration of optimized IPs through suitable communication architecture in short time is desirable. But, it requires comprehensive understanding of functionality and interfaces of each of the IPs. Moreover, no single tool supports seamless modeling and simulation of heterogeneous PEs/IPs with their communication. Mere integration of pre-verified IPs may not meet communication requirements of entire system if designer underestimates communication architecture exploration. At each stage of exploration, quick performance estimation becomes imperative. We strongly believe that SoC design cost and TTM can be reduced if system communication requirements are identified and measures for improvement are introduced early in the design cycle i.e. at the system level. This has been motivation for our efforts for modeling and analysis of communication architecture at the system level of design abstraction.

In this paper, we propose Stochastic Automata Network (SAN) based efficient performance evaluation approach for Network-on-Chip (NoC) architecture. In particular, interaction among concurrent components of these architectures are captured using functional and synchronizing transitions of the SAN model. We use Stateflow component of Matlab [1] to describe SAN models of aforementioned communication architecture. In next section, we present brief background of SAN, and review performance estimation techniques those 
have been proposed in the literature. We propose SAN based modeling framework for NoC architecture in Section 3. In Section 4, we present results. We conclude in Section 5.

\section{Stochastic automata network: An overview}

The behavior of a large, parallel and distributed systems is usually complex and hard to understand as well as to model. Thus, formal techniques are necessary for modeling, verifying and predicting the performance of such systems. A SAN is a powerful formalism, which facilitates the modular description of such systems. In particular, a SAN allows modular description of underlying Markov chain of a system and facilitates computation of the steady state probabilities. These steady state probabilities are required while evaluating performance of the system.

In a SAN, a concurrent system is described as a collection of interacting subsystems. Each subsystem is modeled by a stochastic automaton. Each of the automata consists of a set of states and a set of transitions that determine the dynamic behavior of a component of parallel system. The state of a module is called local state, while global or system state is the collection of local states of all automata. The automata of a SAN model interact with each other using local and synchronizing events. Local event changes the state of a single component automaton by triggering local transition. Synchronizing event modifies the states of more than one module by causing simultaneous transitions in those modules. Probabilities of local and synchronizing transitions can be functional or nonfunctional. In functional transition, transition probability is the function of the states of other modules, whereas it is constant in non-functional transition.

\subsection{Formal description of SAN}

In order to explain formal description of SAN, let us consider a system composed of $\mathrm{N}$ components, and $E$ be a set of synchronizing events with which these component modules interact. The SAN is a set of stochastic automata $\left(A^{(i)}\right)_{i \in[1, \ldots, N]}$. The $i^{\text {th }}$ automaton, $A^{(i)}$ of this set is defined by the tuple $\left(S^{(i)}\right.$.L. $\left.Q^{(i)}\right)$, where $S^{(i)}=\left\{a^{(i)}, b^{(i)}, \ldots\right\}$ is a set of states having cardinality $n_{i} ; L$ is a set of labels; $Q^{(i)}$ is the transition function of automaton and is defined from $S^{(i)} \times S^{(i)} \rightarrow$ $L$. Local state variable of $A^{(i)}$ is denoted by $x^{(i)}$. Hence, global state of SAN is the collection of all local states i.e. a vector $\tilde{x}=\left(x^{(1)}, x^{(2)}, \ldots, x^{(N)}\right)$; whereas $S=S^{(1)} \times S^{(2)} \times \ldots \times S^{(N)}$ is called the global state space with cardinality equal to $\prod_{i=1}^{N} n_{i}$. More details of the SAN model can be found in [2] and [3].

\subsection{Related Work and Our Contribution}

Orgas and Marculescu in [4] proposes analytical performance evaluation for NoC architecture based on router model. The authors assume arbitrary size messages, finite buffers, deterministic routing, round robin router scheduling policy and application-specific traffic patterns. The proposed approach has been used to estimate performance metrics viz. average buffer utilization, average packet latency and network throughput for wormhole flow control. Zhu et al. in [5] propose formal concurrent modeling approach based on operation state machine for entire system comprising of computation and communication for both bus-based and NoC architectures. Authors Pande et al. in their work [6] develop a simulator employing flitlevel, event-driven, wormhole routing for evaluation of performance parameters viz. latency, throughput and energy, while area of NoC architectures has been estimated using synthesized VHDL models. Nandi and Marculescu in [7] use SAN model for system level power/performance analysis in platform based design of heterogeneous architectures. In order to evaluate the power/performance of possible architectures, authors use separate models for application and architectures. Two phase hybrid approaches, which encompass both simulation and analytical techniques have been proposed [8], [9] for performance evaluation of bus-based communication architectures.

Main contribution of this paper lies in proposal for a system level framework based on SAN model for performance estimation of $\mathrm{NoC}$ communication architectures. This model is more comprehensive as compared to models proposed in [4], [6]. We focus on building model for Network-on-Chip (NoC) architecture, and our modeling approach provides evaluation of performance parameters viz. throughput and rate of accepted traffic for mesh, Torus and butterfly Fat Tree topologies.

\section{Proposed SAN based model for an NoC}

In this section, we propose SAN based modeling technique for performance evaluation of NoC communication architecture. Four fundamental characteristics of NoC architectures are following- (i) Network topology e.g. mesh, Torus or butterfly fat tree (BFT); (ii) routing policies e.g. adaptive or non-adaptive; (iii) switching techniques viz. circuit switching, packet switching or wormhole switching; and (iv) flow control methodology to allocate channels and buffers to the flow control units (flits) or packets e.g. fixed priority, round robin or lottery based. The NoC topologies are depicted in Figs. 1, 2 and 3. The multiple levels of 


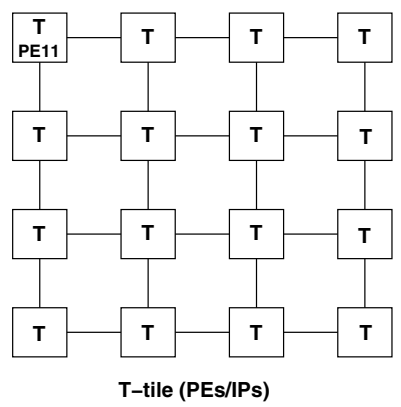

Figure 1. Mesh NoC topology.

concurrency are inherently present in an NoC architecture either at functional level or at architectural level. For example, at the highest level, all the PEs and the routers are concurrent. Further, micro-architecture of each of the routers consists of concurrent buffers and schedulers. The modeling approach significantly changes with the granularity and the level of the concurrency considered. In our work, we assume three functional modules of an NoC architecture viz. PEs, buffers and schedulers function at the same level of concurrency. Channels are used for interconnecting routers as well as for interfacing PEs with the routers. We construct a library of generic blocks that can be combined in a bottom up fashion to model different topologies of NoC. The generic building blocks model different types of resources of NoCs, for example PEs, buffers and schedulers. We instantiate building blocks from the library and integrate various topologies by providing interfaces between blocks. This facilitates a faster exploration of $\mathrm{NoC}$ topologies.

We consider the Torus topology of Fig. 2 to illustrate the SAN modeling technique. For the architecture under consideration, we assume packet switching technique with deterministic $\mathrm{X}-\mathrm{Y}$ routing. Besides, schedulers of the architecture employ lottery based arbitration scheme for allocating output channels to one of the requesting input buffers. For brevity, we illustrate proposed SAN modeling technique for a hop of packet transfer as considered in [5]. Thus, the corresponding SAN model for a hop of NoC architecture has three automata one each for a $\mathrm{PE}$, a buffer and a scheduler. We use notation $P E_{i j}$ for processing elements and $r_{i j}$ for routers of two dimensional Torus architecture, where $i$ and $j$ are the row and column numbers, respectively.

\subsection{Switch architecture}

Figure 5 depicts an architecture of a typical pipelined network router along with its local PE/IP. The router is a five-port pipelined switch with a $5 \times 5$ crossbar. The

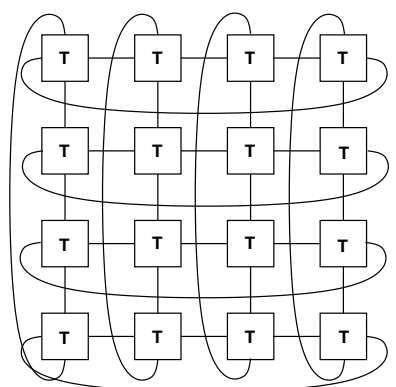

Figure 2. Torus NoC topology.

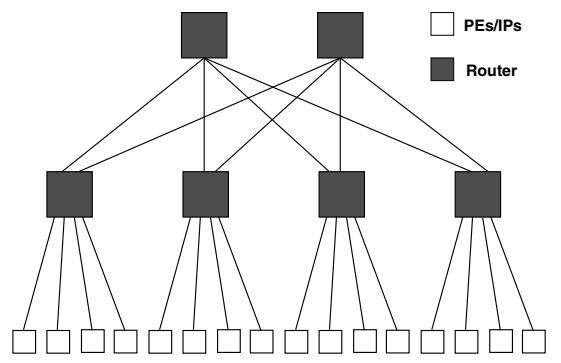

Figure 3. BFT NoC topology.

major components of the router are input controllers, scheduler and crossbar switch. Input controller consists of buffer, routing logic and channel state indication (inp_state). A local PE and neighboring switches can forward packets on input ports of the router if corresponding input buffer is empty. In the buffer, the packets proceed through the states of routing, switch arbitration and switch traversal. When a packet arrives at the input port of the router, the corresponding input buffer stores the packet. Then, the input controller forwards the destination field to the routing logic and sets channel state indication to routing. The routing logic depending on the header of the packet returns the output port for the packet and sets the channel state to switch arbitration. Then, input controller sends a request to the scheduler for a data path inside the crossbar so that the packet can be routed to the desired output port. The scheduler collects all the requests form all the input controllers and grants the access of output ports as per the flow control policy employed. After the scheduler generates grants to the various requests, the packets are sent to the output ports in the next cycle, while channel state indication changes to empty. Thus, in the router the input buffers and the crossbar data paths are two key resources.

\subsection{SAN model of NoC}

In the proposed SAN model, we use three automata to model a hop of the packet transmission within a fiveport pipelined router of the Fig. 5. Our discussion is 


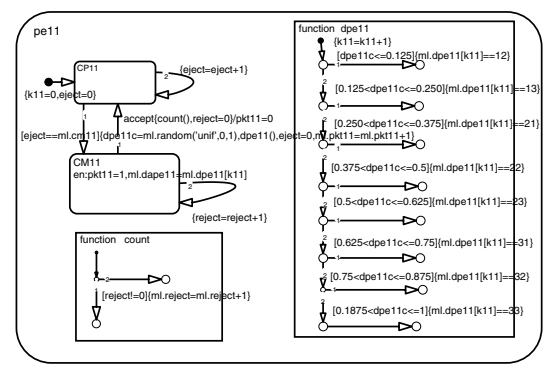

Figure 4. Stateflow structure of a PE.

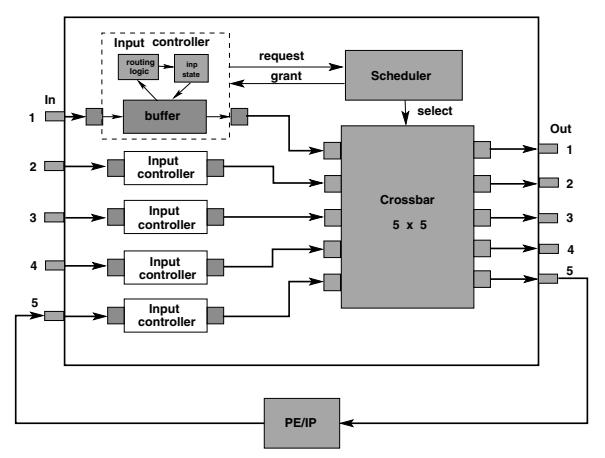

Figure 5. NoC router architecture. this section is focused on the scenario where a hop of packet is transferred from the $P E_{11}$ which is connected to buffer $b 5$ of the router $r_{11}$ to output port two of the router $r_{11}$. Here, $P E_{i j}$ is a processing element connected to router $r_{i j}$ at $i^{\text {th }}$ row and $j^{\text {th }}$ column interjection of mesh/Torus topology. Each of the router $r_{i j}$ consists of five buffers $b_{1}, \ldots, b_{5}$; a scheduler and a crossbar fabric.

The Stateflow toolkit of MATLAB provides an ideal support for the describing the state structure of SAN automata. A Stateflow component is specified in terms of states, state transitions and triggering events. The syntax of the state consist of two major elementsthe name of the state and various state actions viz. entry state action, during state action and exit state action. Moreover, designer can use on event action, which would be executed whenever the specified event occurs. In addition, local and global attributes i.e. data structures can be associated with the Stateflow and with each of the states; whereas state actions can be associated with events and state transitions. The global attributes of the Stateflow model of an SAN automaton $A^{(i)}$ include- (i) a set of synchronizing events $(E)$, (ii) a set of states $(S)$ and (iii) a current global state variable $(\tilde{x})$. Each state has additional local attributes such as local active state $\left(x^{(i)}\right)$, local transitions, random variables and performance indices.

Figure 4 shows Stateflow model of the $P E_{11}$, which has two states computing state, $C P I I$ and communicating state, $C M 11$. A number of computation cycles of the PE are represented by the variable cmll. Alternatively, this variable also specifies rate of traffic ejection of the $P E_{11}$ in the network. In-built Matlab function is used to generate random variable $d p e 11 C$, which is uniformly distributed between zero and one. The function dpe 11 makes a non-deterministic choice of a destination PE depending on the random variable dpellc. The routing information is represented in a shared or global variable dape11[i] for the $i^{\text {th }}$ packet generated by $P E_{11}$, which is being shared among all the automata. Thus, implemented routing is source routing in which routing decision is taken at the source. As packet progresses along the selected path, the content of the variable dape11 [i] will be changed at every hop by the transmitting router till it reaches to the destination PE. The count function uses the global variable reject which returns the the number of packets those have waited at least for one clock cycle before being accepted by the network. The model of the PE transits from the state CPII after computation time of the PE; whereas transition from the state $C M I I$ to the state $C P I I$ is synchronized with the event accept, generated by buffer automaton.

Figure 6 exemplifies the SAN model for present scenario. The automaton, labeled as $A^{P E 11}$, corresponds to the $P E_{11}$ of Torus NoC architecture. Each router has an automaton for each of the buffers and for scheduler corresponding to each of the output ports. Thus, each router of the architecture under consideration has total ten automata. For simplicity in Fig. 6, an automaton for the buffer at input port five, $A^{b 5}-^{r 11}$ and an automaton $A^{\text {sch_o }{ }{ }^{r 11}}$ for the scheduler of the output port two of the router $r 11$ are illustrated.

We model an automaton of a PE as a traffic generator, which generates the packets for various destination PEs in the network. Traffic from a PE to other PEs is uniformly distributed. The destination PE is randomly chosen from a set of intended PEs. The automaton $A^{P E 11}$ has two states computing state labeled as $C P$ and communicating state labeled as $C M$. As soon as a PE completes computation, it generates a packet and transits to communicating state with local transition, labeled as $L$. The transition from communicating state to computing state depends on the network condition, and hence, it is a synchronizing transition with an event $e$ having label $(e, 1,1)$. Here, we use syntax of the label of synchronizing transition i.e. $\left(e, \tau_{e}, p_{e}\right)$ [2], where $e$ is the synchronize transition having event transition probability $\tau_{e}$ and alternate transition probability $p_{e}$. 

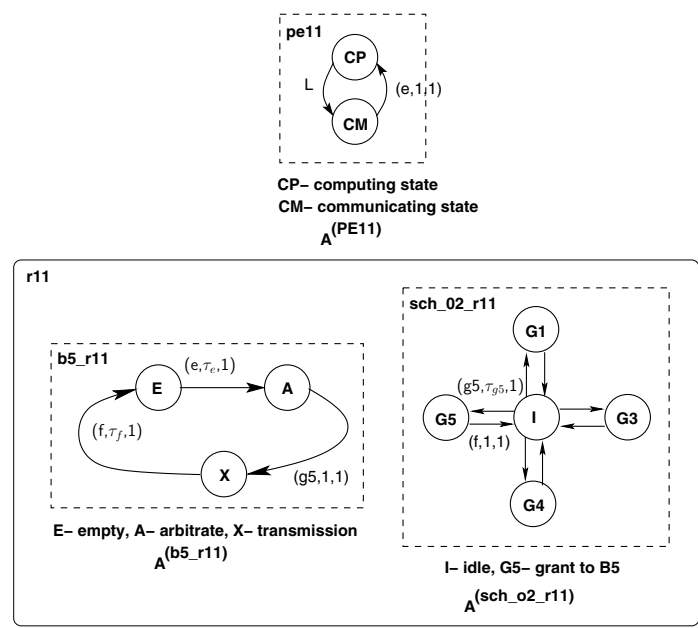

Figure 6. SAN model of NoC architecture.

The label $(e, 1,1)$ in the automaton $A^{P E 11}$ signifies that other automaton decides the event transition probability because $\tau_{e}=1$ in this label. Since there is no alternate transition from the $C M$ state, the label has $p_{e}=1$. The event $e$ is generated in the automaton $A^{b 5}{ }^{r 11}$ when it transits from empty state $(E)$ to arbitration state $(A)$.

Thus, the automata $A^{P E 11}$ and $A^{b 5}-^{r 11}$ make simultaneous transition with synchronize event $e$.

The automaton of the buffer has three states representing three-stage pipelined packet transmissionempty $(E)$, arbitration $(A)$ and transmission $(X)$. Initially, the automaton is in empty state, E. When the packet arrives at the input port of the switch, and if the buffer is in the state $E$, the automaton is activated and progresses from the state $E$ to arbitration state, $A$. In this state, buffer arbitrates for the desired output port by sending request to the corresponding scheduler automaton. The buffer automaton waits in this state for the grant event $(g 5,1,1)$, which is generated by an automaton of the scheduler ( $\tau_{e}=1$ for this event). Note that multiple automata corresponding to other buffers of the same router may be in the state $A$, and may have generated the requests for the same output port in the same clock cycle. Therefore, a scheduler may receive more than one requests from input buffers. Upon receiving the requests, the scheduler decides which request to grant according to its flow control policy. Depending on the granting buffer, automaton $A^{\text {sch_o2_r11 }}$ transits from idle state $I$ to $G 1, G 3, G 4$ or $G 5$, while generating appropriate synchronize grant event. If the scheduler grants request of buffer one, its automaton enters in state $G 1$ and so on. The successful requesting buffer will receive the grant and its automaton proceed to transmission state, $X$. In the state $X$, after one event delay the packet will be transmitted to either the local PE or to any one of the neighboring switches. After the packet transmission, the synchronize event $f$ makes simultaneous transitions from state $X$ to state $E$ in $A^{b 5} r^{r 11}$ and from state $G 1$, $G 3, G 4$ or $G 5$ to state $I$ in $A^{\text {sch_o2 }{ }^{r 11}}$.

\section{Results}

We have estimated performance parameters of SAN based models of NoC architecture. We have formulated high level simulation model based on SAN models in the Stateflow component of Matlab [1]. The simulation was performed on P-IV, 1 GB Linux-WS. We run the Matlab simulation for 10,000 packets from each of the PEs in all considered topologies. Figures 7 and 8 show average values of the estimated parameters.

We have employed the SAN methodology to evaluate throughput and rate of accepted traffic for the three different NoC topologies- Torus, Mesh and BFT. We have used $4 \times 4$ mesh topology, $4 \times 4$ Torus topology and BFT topology with 16 PEs at leaves nodes. In the proposed model, we can choose between uniform and localized traffic patterns for the packets by appropriate selection of destination PEs. The simulator also facilitates the changing of packet generation rate.

Figure 7 illustrates the effect of packets generation rate on the rate of accepted traffic in Torus, mesh and BFT topologies. From the figure, we observe that the accepted traffic increases linearly with the injection load in the beginning and then becomes saturated. We also observe that in Torus topology the networks saturation occurs at slightly higher average data rates i.e. $24 \%$ of injection rate.

An application is generally specified as a set of concurrent tasks, which has been mapped and scheduled onto a set of allocated IP cores for the application. Then, mapping problem for NoCs is to decide how to place and connect various IP cores to the routers of the network. During NoC mapping, objective is to find mapping function such that objective function of energy, latency etc. is optimized. Hence, in the resultant placement more frequent communicating IPs are placed close to each other, making data traffic patterns highly localized. We have studied the effect of traffic localization on throughput. The packets those travel from a source PE to the set of the nearest destination PEs are called local packets. In the Torus architecture, for a PE four destination PEs placed at the shortest distance i.e at one hop are the local PEs; whereas in mesh topology a PE may have two, three or four local PEs. The localization factor is defined as the ratio of local traffic to total traffic. For example, if the four destination PEs are local out of total sixteen destination PEs, the localization factor is 0.25 . It means that 25 percent of the traffic generated by a PE occurs 


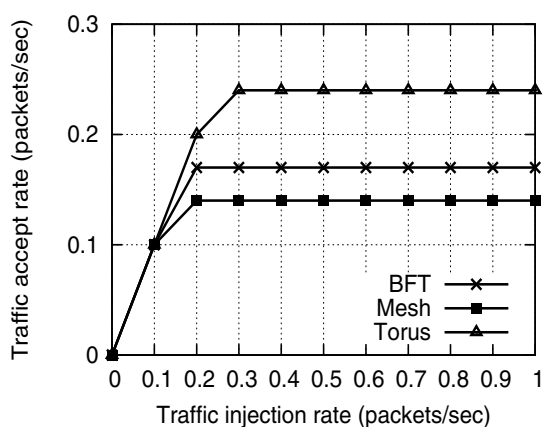

Figure 7. Rate of accepted traffic in NoC topologies.

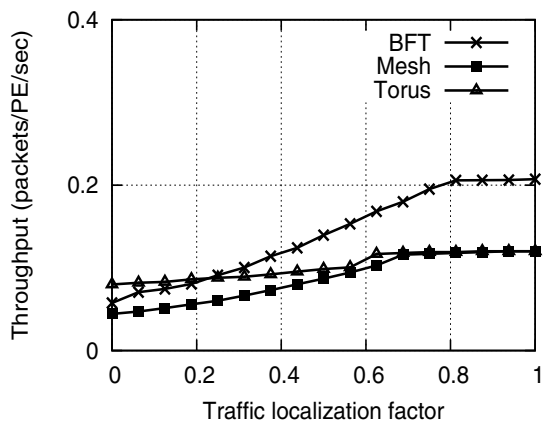

Figure 8. Variation of throughput in various NoC topologies.

within its local group, while the rest of the traffic is randomly distributed in the remainder of the entire SoC.

The throughput variation with respect to traffic localization factor in aforementioned network topologies is illustrated in Fig. 8. As expected, the network throughput increases with the localization factor in all considered topologies. When the traffic is uniformly distributed, the network throughput is higher in Torus topology as compared to mesh and BFT topologies. Whereas for higher localized traffic, network throughput is notable in BFT topology as compared to Torus and mesh topologies. The highest throughput of 0.20 is achieved when the localization factor is $100 \%$ in the BFT topology.

\section{Conclusions}

This paper presents SAN based modeling approach for NoC architecture. We have described the proposed SAN models of this architecture in Stateflow component of Matlab. We have evaluated performance metric viz. throughput and rate of accepted traffic for mesh, Torus and BFT topologies. The developed framework for evaluation is not only efficient and accurate but also requires lesser modeling efforts.

\section{Acknowledgments}

We gratefully acknowledge very helpful suggestions of anonymous reviewers. We also acknowledge financial support provided by Ministry of Communication \& Information Technology, Govt. of India through phase2 of Special Manpower Development Project for VLSI Design \& related manpower.

\section{References}

[1] Stateflow, MathWorks Inc. (2007) [Online] Available: http://www.mathworks. com/products/stateflow.

[2] B. Plateau and K. Atif, "Stochastic automata network for modeling parallel systems," IEEE Tran. on Software Engineering, vol. 17, no. 10, pp. 1093-1108, 1991.

[3] W. J. Stewart, K. Atif, and B. Plateau, "The numerical solution of stochastic automata networks," European Journal of Operation research, vol. 86, no. 3, pp. 503$525,1995$.

[4] U. Ogras and R. Marculescu, "Analytical router modeling for networks-on-chip performance analysis," in Proceedings of IEEE Design Automation \& Test in Europe, April 2007, pp. 1-6.

[5] X. Zhu, W. Qin, and S. Malik, "Modeling operation and microarchitecture concurrency for communication architectures with application to retargetable simulation," IEEE Tran. on VLSI Systems, vol. 14, no. 7, pp. 707716, July 2006.

[6] P. P. Pande, C. Grecu, M. Jones, A. Ivanov, and R. Saleh, "Performance evaluation and design trade-offs for network-on-chip interconnect architectures," IEEE Tran. on Computers, vol. 54, no. 8, pp. 1025-1040, Aug. 2005.

[7] A. Nandi and R. Marculescu, "System-level power/performance analysis for embedded systems design," in Proceedings of IEEE/ACM Design Automation Conference (DAC), 2001, pp. 599-604.

[8] S. Kim, C. Im, and S. Ha, "Schedule-aware performance estimation of communication architecture for efficient design space exploration," IEEE Tran. on VLSI Systems, vol. 13, no. 5, pp. 539-552, May 2005.

[9] K.Lahiri, A. Raghunathan, and S. Dey, "System-level performance analysis for designing on-chip communication architecture," IEEE Tran. on CAD of ICs and Systems, vol. 20, no. 6, pp. 768-783, June 2001.

[10] H. Wang, X. Zhu, L. Peh, and S. Malik, "Orion: A power-performance simulator for interconnection networks," in Proceedings of the 35 th annual ieeelacm international symposium on microarchitecture (MICRO35), 2002. 